\title{
Heartbeat: Colchicine and heart disease
}

\section{Catherine M Otto}

Many lines of evidence suggest that inflammation is a key factor in the pathogenesis of cardiovascular disease so it seems plausible that anti-inflammatory treatment might slow or prevent disease progression. Colchicine is an antiinflammatory medication, originally extracted from the autumn crocus plant, that has been used to treat gout for hundreds of years. Recently there has been interest in whether colchicine might also have cardiovascular benefits. In a Cochrane review and meta-analysis that included 39 trials with a total of 4992 patients with follow-up as long as 14 years, Hemkens and colleagues (see page 590) found no effect on colchicine on all cause mortality or total adverse events (figure 1). However, there was a reduced risk of myocardial infarction with a relative risk of 0.20 (95\% CI 0.07 to 0.57 in 2 trials) at the expense of an increased risk of gastrointestinal intolerance (RR $1.83,1.03$ to 3.26$)$. Results were similar for studies that only included patients with a high cardiovascular risk. Based on this data, the authors estimate that treatment with colchicine for secondary prevention in 1000 patients for 1 year would protect about 20 patients from myocardial infarction, with about 110 patients having mild, transient gastrointestinal symptoms. They conclude: "Colchicine may have substantial cardiovascular benefits; however, there is sufficient uncertainty about its benefit and harm to indicate the need for large-scale trials to further evaluate this inexpensive, promising treatment in cardiovascular disease".

In the accompanying editorial, Imazio and Gaita (see page 569) provide a historical perspective on the use and pharmacology of this agent. As with many anti-inflammatory medications, the exact mechanism of action is unclear with presumed beneficial effects including inhibition of microtubule polymerization, a reduction in mast cell histamine release and blockade of interleukin- $1 \beta$ activation (figure 2).

Imazio and Gaita remind us that "the first use of colchicine in cardiovascular medicine was treatment and prevention of

Correspondence to Professor Catherine M Otto, Division of Cardiology, University of Washington, Seattle,WA 98195, USA; cmotto@u.washington.edu recurrent pericarditis". Now, given the potential benefits of colchicine for a wider range of cardiovascular disease, "we need additional basic and clinical research to better understand the molecular mechanisms, and to verify the real efficacy of colchicine".

The major contributor to a diminished quality of life in patients with chronic heart failure is reduced exercise tolerance. Koshy and colleagues (see page 597) hypothesized that supplemental oxygen would increase exercise capacity by improving oxygen delivery to metabolizing tissues and thus diminishing the sensation of fatigue. Previous studies have shown mixed results and current opinion holds that supplemental oxygen is not beneficial for treatment of patients with chronic heart failure in the absent of hypoxia. In a small, well-designed, randomized, single-blinded, cross-over study of 31 patients with chronic heart failure, Koshy and colleagues found that supplemental oxygen increased exercise time, maximal metabolic equivalents, maximal workload and mean oxygen saturation during exercise (figure 3).

In an editorial, Prior (see page 571) reminds us that "The underlying mechanisms behind the muscle fatigue and dyspnoea which limit exercise capacity in heart failure are very complex, with multiple factors in the chain from the heart and lungs to the muscles contributing to the symptoms. In addition to altered cardiac function and haemodynamics as

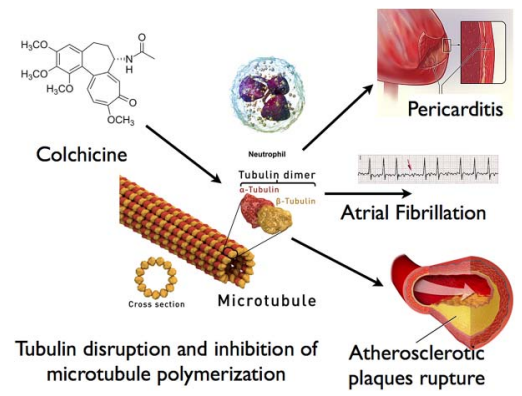

Figure 2 The primary mechanism of action of colchicine is tubulin disruption and thus the inhibition of microtubule polymerisation, an essential component of cellular cytoskeleton. This leads to subsequent potential anti-inflammatory effects especially mediated by its capability to concentrate and act on granulocytes. Such effects in cardiovascular medicine may have application in pericarditis, pericarditis-related atrial fibrillation and atherosclerotic vascular disease.

the central initiator of the heart failure syndrome, abnormalities have been identified in pulmonary function, ventilatory efficiency, peripheral vascular and in particular endothelial function, skeletal muscle structure and skeletal muscle metabolism". Even so, "the work of Koshy suggests that we should not dismiss the possible benefits of oxygen on exercise capacity. The findings need to be reproduced in a study of similar or larger size, we need to understand the mechanism of benefit through more detailed physiological studies of each part in the chain of

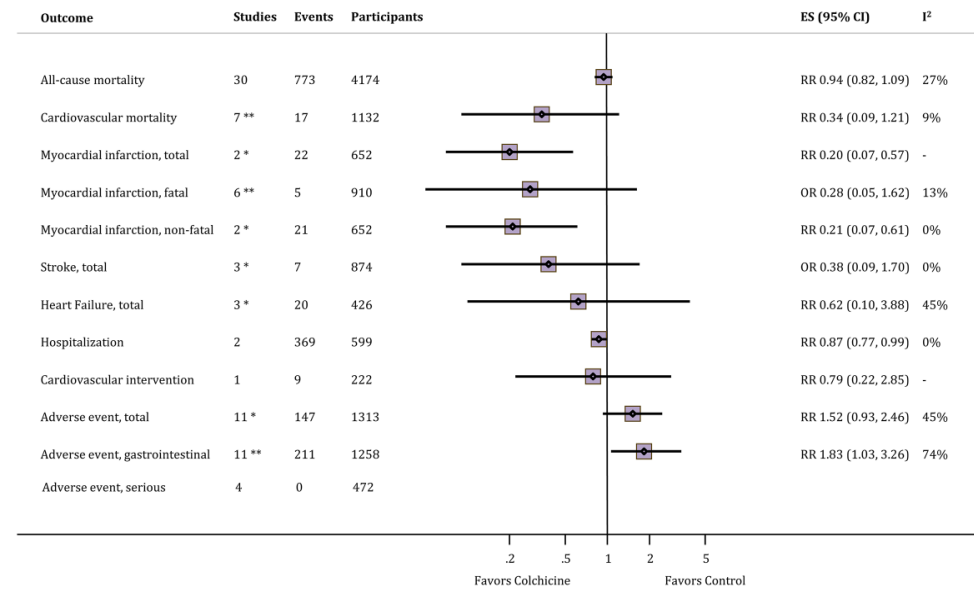

Figure 1 Overview of meta-analyses for colchicine treatment versus control on various patient-relevant outcomes. ES, effect estimate; OR, Peto's OR; RR, relative risk. *Including one study without events. ${ }^{*}$ Including two studies without events. 


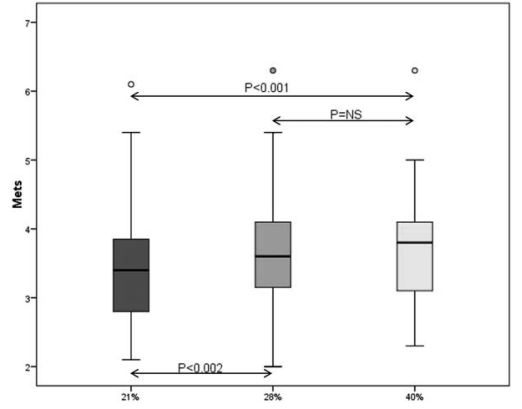

Figure 3 Mean METs increased significantly from room air to $28 \%$ and $40 \%$, respectively, with an almost dose-dependent increase found in median METs. The IQR remains relatively constant with varying $\mathrm{FiO}_{2}$.

oxygen delivery and the effects of chronic oxygen supplementation during exercise need to be explored".

Loeys-Dietz syndrome (LDS), an aortopathy related to mutations in the transforming growth factor $\beta$ receptors 1 or 2 genes, is associated with a high rate of aortic dissection. As we gain clinical experience with this condition, it has become apparent that the range of disease severity and age at clinical presentation can be quite variable. In this issue of Heart, Teixidó-Tura and colleagues (see page 626) extend that clinical experience with an observational report of 58 patients from 11 families with LDS who were followed for a median period of 2.0 years. This patient cohort was older than in previous studies (mean age 42 years) and the first clinical endpoint tended to occur at an older age than previously described: death in $19 \%$ at a median age of 52 years, aortic dissection in 14\% at a median age of 36 years and aortic surgery in $21 \%$ at a median age of 53 years. The rate of aortic dilation, measured in 21 patients, averaged $0.67 \mathrm{~mm} /$ year (IQR $0.16-1.50 \mathrm{~mm} /$ year), with a maximum change of $2.0 \mathrm{~mm} /$ year. Aortic diameter was known in 8 patients before dissection or elective surgery and ranged from 47 to $57 \mathrm{~mm} \mathrm{Hg}$; all of which are much larger than the current recommendations for timing of aortic root replacement in patients with LDS. The high rate of adverse events over a short follow-up interval in this study emphasizes the important of considering genetic testing

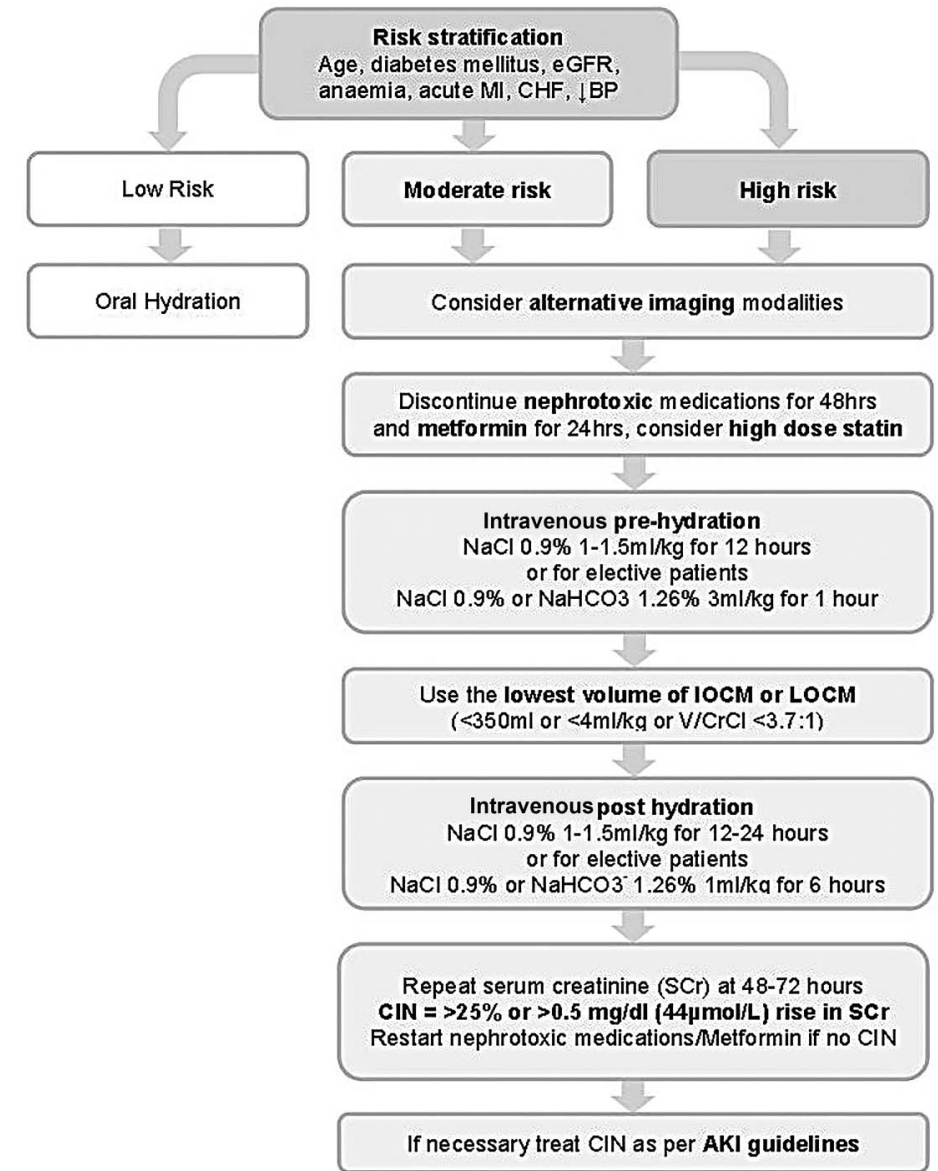

Figure 4 Algorithm for the prevention of $\mathrm{CIN}$ is shown. AKI, acute kidney injury; BP, blood pressure; CHF, chronic heart failure; CIN, contrast-induced nephropathy; eGFR, estimated glomerular filtration rate; IOCM, iso-osmolar contrast medium; LOCM, low-osmolar contrast medium; MI, myocardial infarction; $\mathrm{NaCl}$, sodium chloride; $\mathrm{NaHCO}_{3}^{-}$, sodium bicarbonate; $\mathrm{Scr}$, serum creatinine; $\mathrm{V} / \mathrm{CrCl}$, volume of contrast media to creatinine clearance.

in patients with aortic disease, because the threshold for prophylactic aortic root replacement is a diameter of $5.5 \mathrm{~cm}$ in patients without a genetic aortopathy, $5.0 \mathrm{~cm}$ in those with Marfan syndrome and 4.0 to $4.5 \mathrm{~cm}$ in patients with LDS. ${ }^{1}$

The Education in Heart article (see page 638) in this issue discusses contrast induced nephropathy (CIN) following angiography and cardiac interventions. This article discusses the diagnosis of CIN, clinical outcomes, pathophysiologic mechanisms, risk factors and effective preventative measures (figure 4).

The Image Challenge in the print issue (see page 633) asks you to identify the cause of new ECG changes in an 89 year old man with worsening heart failure.
The online Image Challenge (see page e2) requires you to interpret the parameters summary from an implantable cardioverter defibrillator interrogation in a 69 year old man, illustrating an important concept in management of these patients.

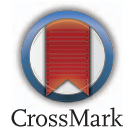

To cite Otto CM. Heart 2016;102:567-568.

Heart 2016;102:567-568.

doi:10.1136/heartjnl-2016-309554

\section{REFERENCE}

1 lung B, New ESC guidelines: aortic disease. Heart. 2015;101:421-3. 